\title{
MHSP with position detection capability
}

\author{
H. Natal da Luz ${ }^{\mathrm{a}, \mathrm{b}}$, J.F.C.A. Veloso ${ }^{\mathrm{a}, \mathrm{b}, *}$, N.F.C. Mendes ${ }^{\mathrm{b}}$, J.M.F. dos Santos ${ }^{\mathrm{b}}$, J.A. Mir ${ }^{\mathrm{c}}$ \\ ${ }^{a}$ Physics Dept., University of Aveiro, 3810-193 Aveiro, Portugal \\ ${ }^{\mathrm{b}}$ Physics Dept., University of Coimbra, 3004-516 Coimbra, Portugal \\ ${ }^{\mathrm{c}}$ CCLRC Rutherford Appleton Laboratory, Chilton, Didcot, Oxon OX11 OQX, UK
}

Available online 20 November 2006

\begin{abstract}
The first implementation of a position sensitive readout for a Micro Hole and Strip Plate (MHSP) is described and tested. The readout consists on a resistive layer crossing the anodes and connected to a preamplifier on each side. By weighing the charge pulses on both preamplifiers it is possible to determine the interaction point. A 100-200 $\Omega$ resistance layer between consecutive strips was found to be the best compromise between position linearity and energy resolution. Preliminary results using $22.1 \mathrm{keV}$ X-rays present a good linearity between the measured and the actual position with a mean deviation of about $0.15 \mathrm{~mm}$ and a position resolution of 1.6 mm full-width at half-maximum (FWHM), being limited by the analogue division electronic circuit. The performance of the MHSP position detector will be presented and discussed for 1D readout.

(C) 2006 Elsevier B.V. All rights reserved.
\end{abstract}

PACS: 29.40.C; 29.40.G; 07.85.F

Keywords: Micro Hole and Strip Plate; Gas Electron Multiplier; X-ray detector; Position sensitive; Resistive charge division; Analogue division

\section{Introduction}

X-ray imaging has become a very useful tool in many branches of science. It is of major importance for analysis and diagnosis in biology and medicine, but its use is also increasing in neutron applications. The need of gas detectors with high gains and good stability at high pressure has put the novel Micro Hole and Strip Plate (MHSP) [1] in a privileged position for its development for neutron detection [2]. The MHSP has an intrinsic capability for position detection [1]. This gaseous electron multiplier is conceived as a combination of a Gas Electron Multiplier (GEM) and a Micro Strip Gas Chamber (MSGC) in a single, double sided element, integrating two successive independent stages of charge amplification: a GEM-like hole-avalanche and an MSGC-like anode-strip avalanche. Like the GEM, the MHSP is fabricated with

\footnotetext{
${ }^{*}$ Corresponding author. Physics Dept., University of Aveiro, 3810-193 Aveiro, Portugal.

E-mail address: jveloso@fis.ua.pt (J.F.C.A. Veloso).
}

printed circuit board (PCB) technology from a $50-\mu \mathrm{m}$ Kapton ${ }^{\mathrm{TM}}$ film, metallized with 5 - $\mu$ m-thick copper-layers on both sides. On the top-side, a GEM-like pattern of holes is etched through, where on the bottom side a standard microstrip pattern is etched, with the holes centered on the cathode strips. The MHSP achieves gains in excess of $10^{4}$ in a Xenon atmosphere [3], and a ratio between top and anode signals of about 0.35 , allowing measuring the charge signal produced on both sides with good efficiency.

In this work, a special MHSP was designed with independent anodes to measure 1D X-ray interaction position. A resistive layer was placed, by serigraphy deposition, connecting all the anodes, Fig. 1. The signals produced by the incoming radiation were collected from both ends of this resistance. The method of resistive charge division is the most simple and inexpensive to implement in this type of microstructures. Delay lines [4] or independent readout of the anodes by high-density electronics [5] are not compatible with the objective of building a simple, lowcost position sensitive gas detector, such as that proposed here. Furthermore, resistive charge division for position 


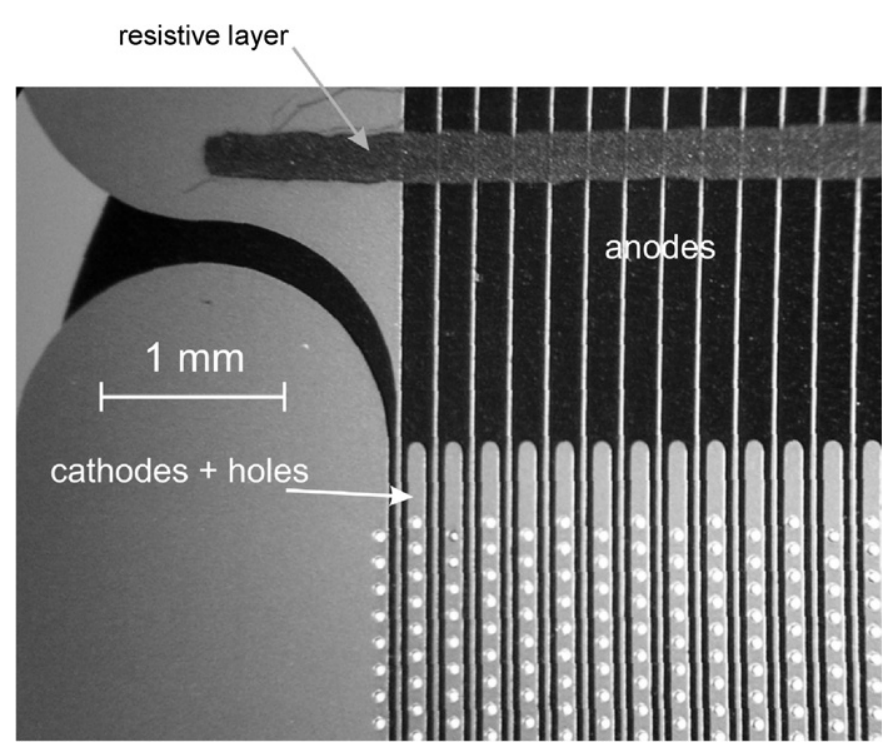

Fig. 1. The bottom side of the MHSP adapted for position readout, with the resistive layer crossing all the independent anodes.

detection has already been successfully used in MSGC [6,7], with spatial resolutions of $1.2 \mathrm{~mm}$ for neutron detection.

\section{Experimental setup}

The working principle of the MHSP can be found in Ref. [1-3, and references therein]. Fig. 1 shows a detail of the bottom face of the structure developed for this work, where the perforated cathodes, the independent anodes and the new resistive layer can be seen. The cathodes are $100 \mu \mathrm{m}$ wide and the pitch is $200 \mu \mathrm{m}$. The distance between the holes in the cathode direction is $140 \mu \mathrm{m}$. The detection area is $28 \times 28 \mathrm{~mm}^{2}$.

The best value for the resistance between each anode provides a compromise between position linearity with minimization of side effects and acceptable $S / N$ ratio. This value was determined by dividing the charge collected from a standard proportional counter-simulating one single anodethrough different resistor chains, and plotting the simulated position. The best value was found to be between 100 and $200 \Omega$ per anode strip, which means around $20 \mathrm{k} \Omega$ in total.

The MHSP was placed $5 \mathrm{~mm}$ from the $25 \mu \mathrm{m}$ thick Mylar $^{\mathrm{TM}}$ window of the detector and $3 \mathrm{~mm}$ from the induction plane at the bottom. The detector filling gas was Xenon, purified through SAES Getters.

The charge produced by the detector is collected by the two preamplifiers connected to the left and right ends of the resistive layer. The position $X$ of the interaction is derived by

$X=k \frac{X_{\mathrm{L}}}{X_{\mathrm{L}}+X_{\mathrm{R}}}$,

where $X_{\mathrm{L}}$ and $X_{\mathrm{R}}$ are the amplitudes of the pulses collected from the left and the right sides of the resistive layer, respectively and $k$ is a calibration constant.

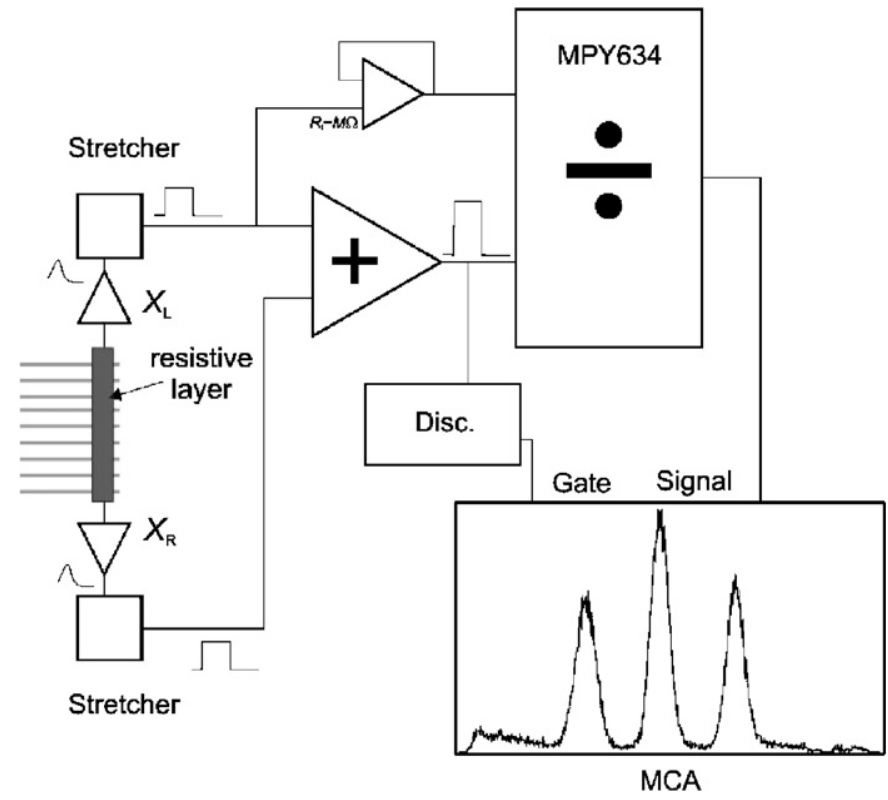

Fig. 2. The low-cost analogue circuit used in this work for the position readout.

The readout was made using Canberra 2006 charge preamplifiers and Tenelec 243 amplifiers with shaping time of $0.5 \mu$ s. Fig. 2 shows the processing electronics based on standard components, including a low cost analogue divider MPY634. This layout is similar to that used in Ref. [7]. Since there is a maximum time difference of about $100 \mathrm{~ns}$ between the arrival of both pulses to the amplifiers, they were stretched in order to achieve a better accuracy when summing and dividing.

For the measurements, the $\mathrm{Ag} \mathrm{K}_{\alpha}$ and $\mathrm{K}_{\beta} \mathrm{X}$-rays of a ${ }^{109} \mathrm{Cd}$ source were used to irradiate the detector window through five different holes, $2 \mathrm{~mm}$ in diameter, of a $3 \mathrm{~mm}$ thick stainless steel collimator. The holes are separated $6 \mathrm{~mm}$ from each other, over a width of $24 \mathrm{~mm}$, and aligned perpendicularly to the MHSP strips. A discriminator was used to reject the pulses due to X-ray fluorescence from the copper present in the MHSP, Xe escape peaks and electronic noise. The TTL pulses arising from the discriminator provide the gate signal for the ADC of the multi-channel analyzer.

\section{Experimental results and discussion}

To evaluate the position linearity, separated measurements were made in the five holes of the detector window collimator. Fig. 3 shows the relationship between the position of the X-ray source and the correspondent measured position using Eq. (1). The peaks were measured separately and were normalized. On the left- and rightmost positions the peaks are distorted because they are located at the borders, which correspond to the limits of the $28 \mathrm{~mm}$ detection width of the MHSP.

The right side of the graphic shows the difference between the X-ray source position and the measured 


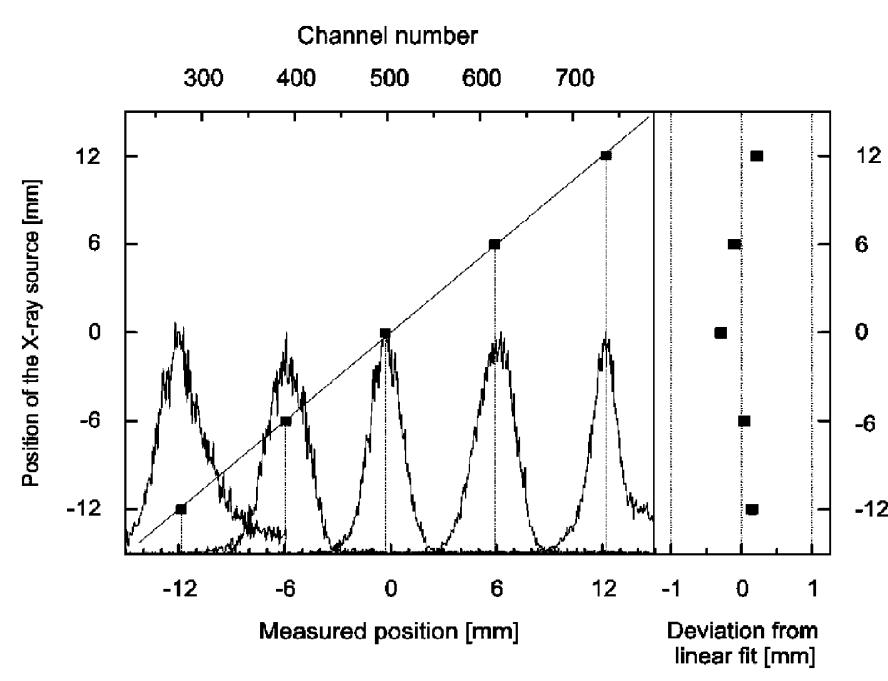

Fig. 3. Position linearity of the system and deviation from linearity trend line.

position. The mean of the absolute value of this difference is $0.15 \mathrm{~mm}$. This demonstrates good position linearity, and the image distortion caused by this deviation is small compared with the achievable position resolution.

The peaks shown in Fig. 4 were obtained with the X-ray source placed $5 \mathrm{~cm}$ above the detector window. At the best measuring conditions, the peaks are totally separated from each other due to its spatial resolution and low background. The higher intensity of the central peak reveals that the source was placed at the center position. Due to the above-mentioned distortions the peaks at the borders are not shown in this graphic. The full-width at halfmaximum (FWHM) of the central peak is lower than the $2 \mathrm{~mm}$ of the hole because we have used circular holes instead of slits, meaning that only at the center the hole width is $2 \mathrm{~mm}$. The other peaks show slightly higher FWHM due to the fact that for these positions the X-rays do not enter the window perpendicularly.

When a smaller collimator was used $(0.5 \mathrm{~mm}$ diameter hole) no improvements in spatial resolution were noticed. To test if the limitations in resolution are due to the detector or else, to the analogue division circuit, a BNC pulse generator was connected directly to the inputs of both shaping amplifiers, substituting the detector and charge pre-amplifiers. It was noticed that, for each constant pulse height, the degradation in the position resolution introduced by the processing electronics is negligible. However, for an increase of about $10 \%$ in height of the signal output in both shaping amplifiers (which is the typical detector energy resolution for $22.1 \mathrm{keV}$ in this experimental conditions) a position variation of 30 channels was observed, corresponding to about $1.3 \mathrm{~mm}$. This suggests that the spatial resolution of the detector is being limited by the accuracy of the analogue division circuit, which has a pulse height-dependent behavior. This confirms the need for upgrades made in recent models of analogue devices, which are no longer sensitive to variable denominator voltages [8]. These upgraded devices will be

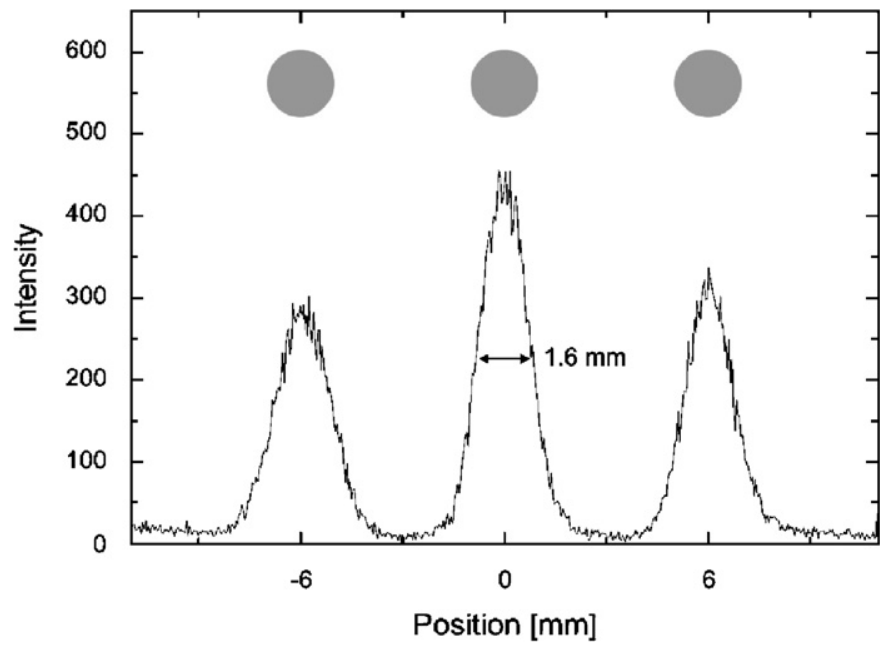

Fig. 4. X-ray position when the ${ }^{109} \mathrm{Cd}$ source is irradiating the three central holes of the window from a distance of $5 \mathrm{~cm}$.

part of the next analogue division circuit to minimize this effect, and fully exploit the position detection capabilities of this detector.

\section{Conclusion}

The 1D position detection capability of the MHSP operated with a resistive layer charge division was investigated. A good position linearity was achieved, while the position resolution was limited to $1.6 \mathrm{~mm}$. The present studies have shown that this limitation is due to the poor accuracy achieved by the analogue division electronics and not to the detector.

Future work will aim an improved low-cost electronic readout that introduces a negligible contribution to the intrinsic position resolution of the detector. In addition, a new MHSP with 2D position capability, having a structured top surface with strips perpendicular to those on the bottom surface [1], using resistive charge division is already in preparation.

\section{Acknowledgments}

This work was supported by project POCTI/FP/FNU/ 50218/2003 through FEDER and FCT (Lisbon). H. Natal da Luz acknowledges support from FCT and from PSD7 Organizing Committee to participate in the conference. We would like to thank Drs. Nigel Rhodes and Erik Schooneveld of the ISIS facility at the CCLRC Rutherford Appleton Laboratory for their continuous interest and support during these studies.

\section{References}

[1] J.F.C.A. Veloso, J.M.F. dos Santos, C.A.N. Conde, Rev. Sci. Instrum. $71(2000) 2371$.

[2] J.F.C.A. Veloso, F.D. Amaro, J.M.F. dos Santos, J.A. Mir, G.E. Derbyshire, R. Stephenson, M.J. Rhodes, E.M. Schooneveld, IEEE Trans. Nucl. Sci. NS-51 (2004) 2104. 
[3] H. Natal da Luz, J.F.C.A. Veloso, F.D. Amaro, L.F. Requicha Ferreira, J.M.F. dos Santos, A. Breskin, R. Chechik, Nucl. Instr. and Meth. A 552 (2005) 259.

[4] G.P. Guedes, A. Breskin, R. Chechick, D. Vartsky, D. Bar, A.F. Barbosa, P.R.B. Marinho, Nucl. Instr. and Meth. A 513 (2003) 473.

[5] A. Bressan, R. de Oliveira, A. Gandi, J.-C. Labé, L. Ropelewski, F. Sauli, D. Mörmann, T. Müller, H.J. Simonis, Nucl. Instr. and Meth. A 425 (1999) 254.
[6] N. Vellettaz, J.E. Assaf, A. Oed, Nucl. Instr. and Meth. A 392 (1997) 73.

[7] J.E. Bateman, J.F. Connolly, A.B. Lodge, R. Stephenson, R. Mutikainen, I. Suni, J. Morse, Nucl. Instr. and Meth. A 477 (2002) 29.

[8] Analog Devices, AS734, 10 MHz 4-Quadrant Multiplier/Divider, Data Sheet, available in: 〈http://www.alldatasheet.net/datasheet-pdf/pdf/ 48194/AD/AD734.html $\rangle$. 